\title{
Dependence of fluxon dynamics on loaded terminations in long overlap Josephson junctions
}

\author{
Olsen, O.H.; Samuelsen, Mogens Rugholm
}

Published in:

Journal of Applied Physics

Link to article, DOI:

10.1063/1.332307

Publication date:

1983

Document Version

Publisher's PDF, also known as Version of record

Link back to DTU Orbit

Citation $(A P A)$ :

Olsen, O. H., \& Samuelsen, M. R. (1983). Dependence of fluxon dynamics on loaded terminations in long overlap Josephson junctions. Journal of Applied Physics, 54(5), 2777-2780. https://doi.org/10.1063/1.332307

\section{General rights}

Copyright and moral rights for the publications made accessible in the public portal are retained by the authors and/or other copyright owners and it is a condition of accessing publications that users recognise and abide by the legal requirements associated with these rights.

- Users may download and print one copy of any publication from the public portal for the purpose of private study or research.

- You may not further distribute the material or use it for any profit-making activity or commercial gain

- You may freely distribute the URL identifying the publication in the public portal 


\title{
Dependence of fluxon dynamics on loaded terminations in long overlap Josephson junctions
}

\author{
O. H. Olsen \\ NIRO Atomizer, Research Development System and Design Division, Gladsaxevej, DK-2860 Soeborg, \\ Denmark
}

M. R. Samuelsen

Physics Laboratory I, The Technical University of Denmark, DK-2800 Lyngby, Denmark

(Received 23 August 1982; accepted for publication 6 October 1982)

\begin{abstract}
Fluxon motion in long overlap-geometry Josephson junctions influenced by loaded terminations is investigated. The combination of results from a perturbation method with an approximate expression for the emitted power yields equations describing the fluxon dynamics. The form of the first zero field step is presented for various parameters and the emitted power is calculated.
\end{abstract}

PACS numbers: 85.25. $+\mathrm{k}, 74.50 .+\mathrm{r}, 84.20 . \mathrm{Pc}, 84.30 . \mathrm{Ng}$

\section{INTRODUCTION}

Recently, fluxon (i.e., the quantized magnetic vortices present in long Josephson junctions) dynamics have attracted considerable interest because of the many potential applications of Josephson junctions, e.g., in data processing systems ${ }^{1}$ and as microwave generators in high frequency integrated circuits. ${ }^{2}$ The latter application arises because the fluxons propagating in a dissipative and biased junction attain steady states determined by power balance. Thus, the fluxons regain from the bias the energy they lose when reflected at lossy terminations. In such oscillator applications, the power radiated from the junction is determined by the resistive terminations. In Ref. 3, resonant fluxon propagation on an overlap-geometry junction with a variable resistive load at one end is studied numerically for a specific choice of loss and bias parameters, and the optimum load resistance for maximum power is determined. This investigation and many others like $\mathrm{it}^{4}$ have been carried out by a numerical solution of perturbed sine-Gordon equations which model the problem in order to obtain general knowledge about the dependence of basic dynamic proporties of single fluxons on external parameters. Recently, ${ }^{5}$ however, it has been proved possible to calculate the shape of the zero field steps in an inline junction using a perturbation theory. The same idea was used to calculate the dependence of zero field steps on an external magnetic field in inline as well as overlap geometry. ${ }^{6}$ In the present paper we apply a method similar to that in Ref. 6 to calculate the dependence on resistive terminations of the $I-V$ characteristics and the emitted power from the junction. We only consider the first zero field step of overlap-geometry Josephson junctions. The expression for the radiated power as a function of the velocity of the incoming fluxon and the resistive load found in Refs. 7 and 8, is used in connection with perturbation results to combine the effects of resistive loads at each termination and the presence of loss and bias in the junction.

\section{MODEL}

In this section we shortly describe the equation which models the overlap-geometry junction and the boundary conditions for this equation which model the resistive terminations.

\section{A. Overlap-geometry junction}

It is well known that the equation describing fluxon motion for this geometry is the perturbed sine-Gordon equation $^{9}$

$$
\phi_{x x}-\phi_{t t}-\sin \phi=\alpha \phi_{t}+\eta,
$$

where $\phi(x, t)$ is the space and time dependent phase difference between the two superconducting films. The spatial variable $x$ is measured in units of the Josephson penetration depth $\lambda_{J}=\left(\hbar / 2 \mu_{0} e d J\right)^{1 / 2}$ and time $t$ in units of the reciprocal plasma frequency $\omega_{0}^{-1}$, where $\omega_{0}=(2 e J / \hbar C)^{1 / 2}$. Here, $J$ is the maximum pair current density, $d$ the magnetic thickness of the barrier $\left(d=2 \lambda_{L}+t_{0}\right)$, and $C$ the capacitance per unit area. The parameter $\alpha$ describes dissipative effects; $\alpha=G(\hbar / 2 e J C)^{1 / 2}$, where $G$ is the shunt conductance per unit area. $(\alpha=1 / \sqrt{\beta}$, where $\beta$ is the usual McCumber parameter.) $\eta$ represents the uniformly distributed bias current; thus, $\eta=I_{\mathrm{dc}} / I_{\mathrm{c}}$, where $I_{\mathrm{dc}}$ is the dc bias current; $I_{c}=J W L$ is the maximum supercurrent, and $L$ and $W$ are the length and width of the junction. It is assumed that $L \gg \lambda_{J}>W$.

\section{B. Resistive terminations}

The voltage drop across the insulating barrier in the junction is $\phi_{t}$ measured in units of $\hbar \omega_{0} / 2 e$. The current flowing parallel to barrier in the $x$ direction is $-\phi_{x}$ measured in units of $J \lambda_{J}$. Thus, the boundary conditions which must be satisfied are

$$
\phi_{t}(0, t)=R \phi_{x}(0, t)
$$

and

$$
\phi_{t}(l, t)=-R \phi_{x}(l, t) .
$$

Here, the terminal resistance $R$ is normalized to the characteristic impedance of the junction $\left(\mu_{0} d / c\right)^{1 / 2}$ and $l=L / \lambda_{J}$.

With $\alpha$ and $\eta$ equal to zero Eq. (1) is the pure sineGordon equation with the fluxon (soliton) solution 


$$
\phi(x, t)=4 \tan ^{-1}\left\{\exp \left[\gamma(u)\left(x-u t-x_{0}\right)\right]\right\},
$$

where $x_{0}$ is the initial position of the fluxon with velocity $u$, and the Lorentz factor $\gamma(u)=\left(1-u^{2}\right)^{-1 / 2}$. The energy absorbed in $R$ after reflection of a fluxon into an antifluxon was found in Ref. 7 to be approximately

$$
\Delta H=\frac{2}{R}\left\{\frac{H_{j}^{2}}{8} \tan ^{-1} \frac{8}{P_{j}}+\frac{P_{j}}{8}\right\}, \quad R \gg 1 .
$$

Here, $H_{j}$ is the energy of the incoming fluxon

$$
H_{j}=8 \gamma(u) \text {, }
$$

and $P_{j}$ is the momentum of the incoming fluxon

$$
P_{j}=8 u \gamma(u) \text {. }
$$

In the following, $\Delta H$ from Eq. (4) will be used as a measure of the emitted power from the junction since the velocity is almost constant.

\section{PERTURBATION THEORY}

It is a well known result that the steady state velocity $u_{\infty}$ is obtained in the presence of loss and bias $\alpha \phi_{t}$ and $\eta$, respectively. For $\eta$ and $\alpha \phi_{t}$ treated as small perturbations $u_{\infty}$ (normalized to $\lambda_{j} \omega_{0}$ ) is given by ${ }^{9}$

$$
u_{\infty}=\left(1+\left(\frac{4 \alpha}{\pi \eta}\right)^{2}\right)^{-1 / 2} \text {. }
$$

With a fluxon (antifluxon) reflected as an antifluxon (fluxon) at the junction terminations this gives rise to a zero field step at a voltage $V_{\mathrm{dc}}=\left(2 \pi u_{\infty}\right) / l$ normalized to $\hbar \omega_{0} / 2 e$. The boundary conditions of Eq. (2) give rise to emission of power given by Eq. (4).

Figure 1 shows schematically fluxon dynamic behavior with an energy output at $x=0$, and a rise in velocity towards $u_{\infty}$ as it approaches $x=l$ where there is a velocity (energy) decrease. Thus, the picture is symmetric (because of the equal loads at $x=0$ and $x=l$ ).

In order to calculate those trajectories we introduce the following notation ${ }^{6}$ for convenience:

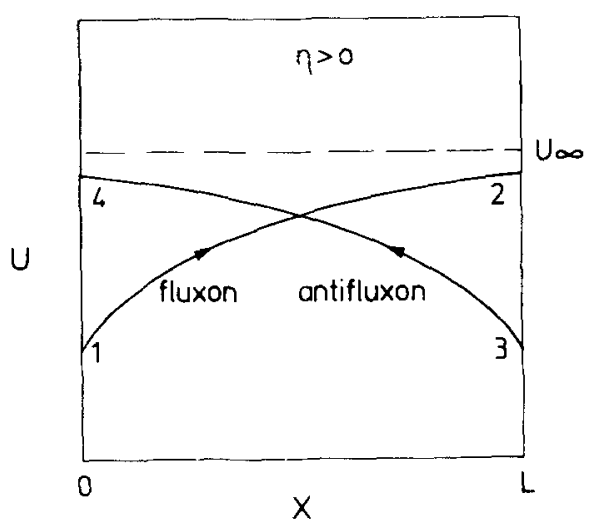

FIG. 1. Schematic picture of the periodic fluxon velocity vs position $x$ in the overlap-geometry Josephson junction $(0 \leqslant x \leqslant L)$. The picture is symmetric due to equal loads at the terminations. At $x=L$ the fuxon is reflected as an antifuxon with less energy. From 3 to 4 the antifluxon accelerates towards the velocity $u_{\infty}$. At $x=0$ the antifluxon is reflected into a fluxon and the behavior is as for the antifluxon.

$$
\begin{aligned}
& u=\tanh a, \\
& z=u \gamma(u)=P_{f} / 8,
\end{aligned}
$$

which implies that

$$
\begin{aligned}
& \gamma(u)=\cosh a, \\
& z=\sinh a, \\
& z_{\infty}=\pi \eta / 4 \alpha, \\
& u_{\infty}=\tanh \mathrm{a}_{\infty} .
\end{aligned}
$$

The equation of motion for a single fluxon in the perturbed sine-Gordon model is ${ }^{9}$

$$
\frac{d P_{f}}{d t}=-\alpha P_{f}+2 \pi \eta \text {. }
$$

Introducing the quantity $z$ from Eq. (9) and integrating we obtain

$$
z=z_{\infty}+\left(z_{0}-z_{\infty}\right) e^{-\alpha t}
$$

where $z_{0}=u_{0} \gamma\left(u_{0}\right), u_{0}$ being the initial velocity of the fluxon. Finding $u(t)$ from Eqs. (8) and (11) integrating yields the trajectory $x(t)$

$$
\begin{aligned}
x(t)= & x_{0}+u_{\infty} t-\frac{1}{\alpha} \ln \frac{z+\left(z^{2}+1\right)^{1 / 2}}{z_{0}+\left(z_{0}^{2}+1\right)^{1 / 2}} \\
& -\frac{u_{\infty}}{\alpha} \ln \frac{1+z_{\infty} z_{0}+\left(z_{\infty}^{2}+1\right)^{1 / 2}\left(z_{0}^{2}+1\right)^{1 / 2}}{1+z \cdot z_{0}+\left(z^{2}+1\right)^{1 / 2}\left(z_{0}^{2}+1\right)^{1 / 2}}(1)
\end{aligned}
$$
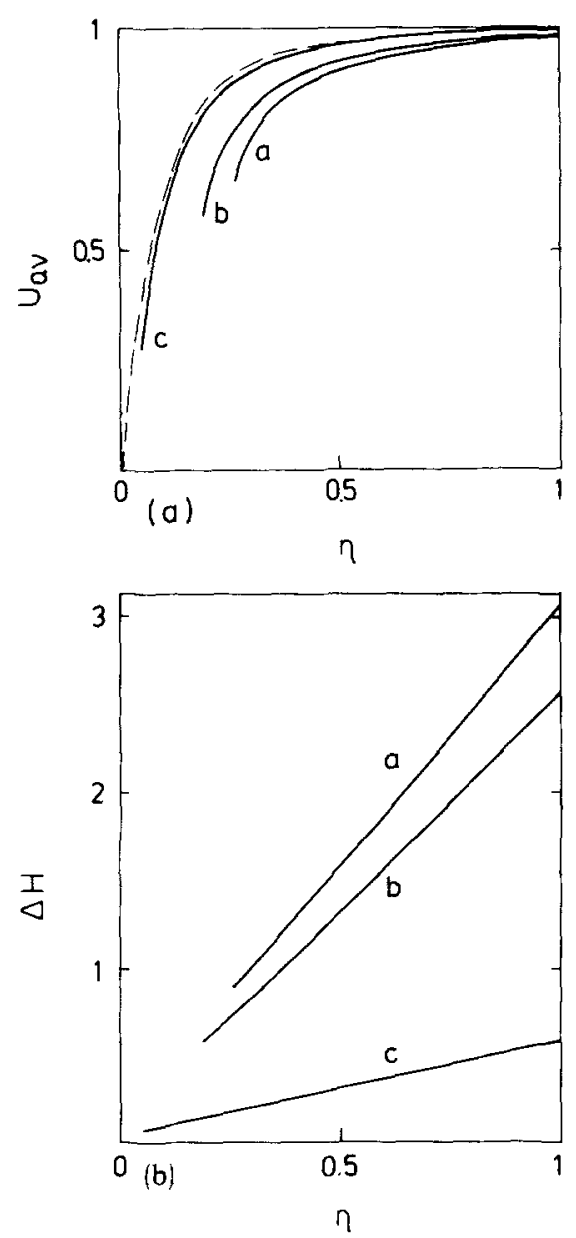

FIG. 2. Average velocity $u_{a v}$ (voltage) (a) and the emitted power $\Delta H$ (b) vs the bias parameter (dc current) at various loads for the first zero field step. $\alpha=0.1$ and $l=10 . R=$ (a) 8, (b) 10 , and (c) S0. The dashed curve in 2(a) corresponds to $u_{a v}=u_{\infty}$, i.e., $R=\infty$. Note the cutoff at finite current. 

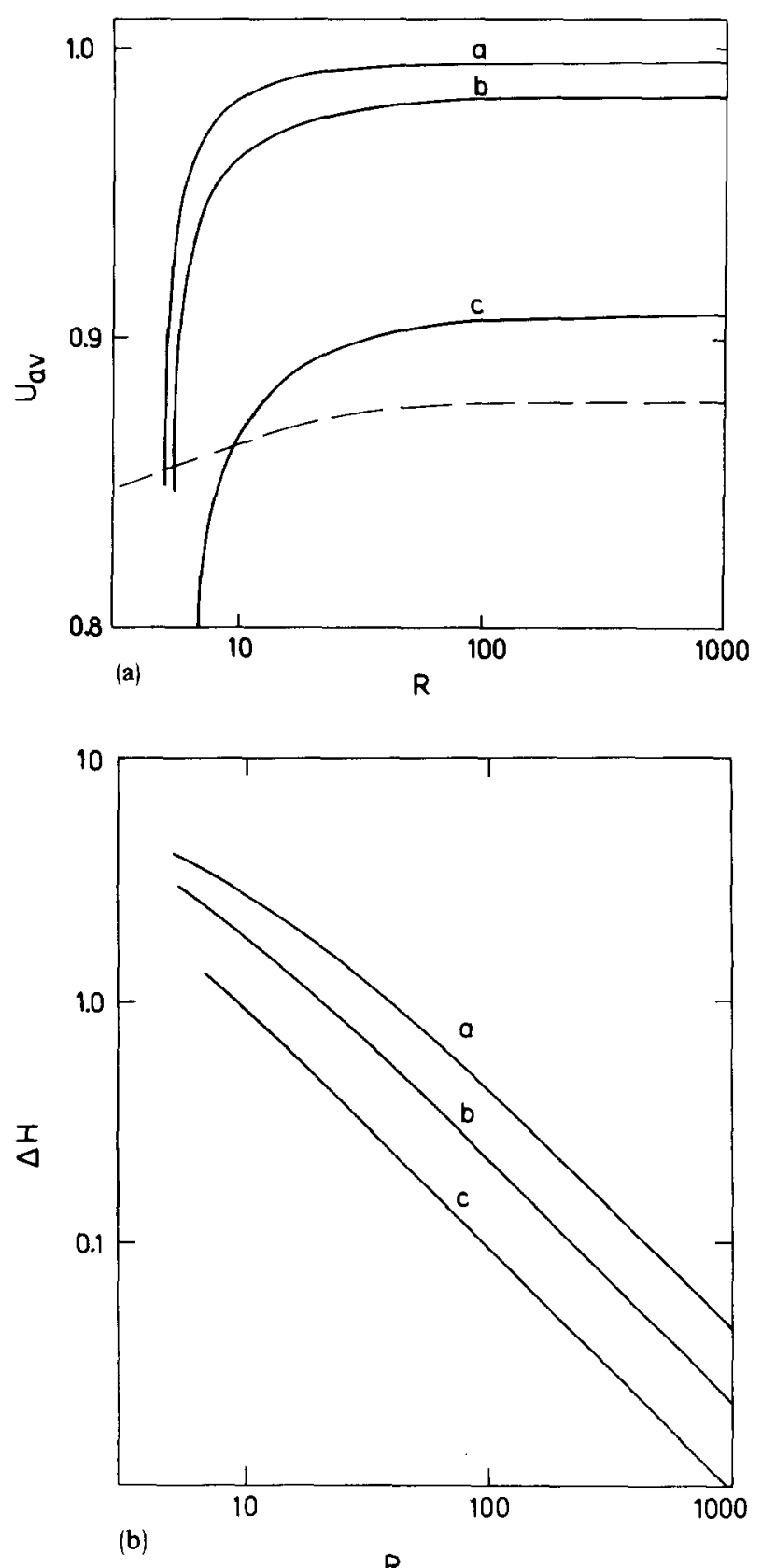

FIG. 3. Average velocity (voltage) (a) and the emitted power $\Delta H$ (b) vs the terminating load at various values of the loss parameter $\alpha . \eta=0.7$ and $l=10 . \alpha=(a) 0.05$, (b) 0.1 , and (c) $0.2523\left[=(5 \pi)^{-1 / 2}\right]$. The dashed curve in (a) corresponds to results obtained by numerical solution of the perturbed sine-Gordon equation.

With reference to Fig. 1 and Eq. (4) the energy output at $x=l$ and $x=0$ may be expressed

$$
8 \gamma\left(u_{4}\right)-8 \gamma\left(u_{1}\right)=8 \gamma\left(u_{2}\right)-8 \gamma\left(u_{3}\right)=\Delta H,
$$

or

$$
\begin{aligned}
& \frac{2}{R}\left\{\left(\cosh a_{j}\right)^{2} \tan ^{-1} \frac{1}{\sinh a_{j}}+\sinh a_{j}\right\} \\
& =\cosh a_{j}-\cosh a_{i} ; \quad i, j=\begin{array}{l}
1,4 \\
3,2
\end{array}
\end{aligned}
$$

Because of the symmetry the two equations [Eqs. (13a) and (13b)] are identical. (11)

The times of flight $t_{12}$ and $t_{34}$ are determined from Eq.

$$
t_{12}=t_{34}=\ln \frac{\sinh a_{4}-\sinh a_{\infty}}{\sinh a_{1}-\sinh a_{\infty}} .
$$

The equation introducing the length of the junction comes from Eq. (12):

$$
\frac{a l}{u_{\infty}}=\alpha t_{12}-\frac{a_{1}-a_{4}}{u_{\infty}}+2 \ln \frac{\cosh \left(\frac{a_{4}+a_{\infty}}{2}\right)}{\cosh \left(\frac{a_{1}+a_{\infty}}{2}\right)} .
$$

Now we have two equations, Eqs. (13) and (15), to determine the two unknowns $a_{1}\left(=a_{3}\right)$ and $a_{4}\left(=a_{2}\right)$ which, inserted in Eq. (14), gives $t_{12}\left(=t_{34}\right)$. The voltage of the zero field step is given by the average velocity $u_{a v}$ defined by $u_{a v}=l / t_{12}$ $\left(=l / t_{34}\right)$. Because of the complexity of the expressions [Eqs. (13)-(15)] we have not been able to find a closed expression for this quantity. However, a numerical solution of the equations has been performed (using Newton-Raphson iteration). The results are presented in the next section in the form of $I-V$ characteristics and emitted power versus load characteristics.

\section{I-V CHARACTERISTICS AND EMITTED POWER}

In Fig. 2 we show the $I-V$ characteristics and the emitted power. Figure 2(a) shows the $I-V$ characteristics, i.e., the average velocity $u_{a v}$ (voltage) versus the bias parameter (dc current) at various loads for the first zero field step. The dotted curve corresponds to $u_{a v}=u_{\infty}$; i.e., $R=\infty$. Note the cutoff at finite current which is also observed in laboratory experiments as well as numerical experiments. ${ }^{4}$ In our analysis the cutoff corresponds to absorption of the fluxon at a termination, while Lomdahl ${ }^{4}$ has found a transition to a static solution in numerical experiments for small bias values. Furthermore, our analysis does not reveal the transition from single fluxon propagation to uniform $\phi$ excitation which in the $I-V$ curve corresponds to a jump from the zero field step to the ohmic background. In Fig. 2(b) we show the emitted power as a function of the dc bias current for the same values of the loads as in Fig. 2(a). The dashed curve in Fig. 2(a) corresponds to $\Delta H=0$ in Fig. 2(b). The results are in qualitative agreement with experiments; i.e., the power increase is almost linear for increasing bias.

In Fig. 3 we show the average velocity (voltage) [Fig. 3(a)] and the emitted power $\Delta H$ [Fig. 3(b)] versus the terminating load at various values of the loss parameter $\alpha$. The dotted curve in Fig. 3(a) corresponds to a numerical experiment performed in Ref. 3 for the same parameters as in case $c$ of Fig. 3. The agreement is within a few per cent; although, $\alpha$ and $\eta$ are large. Figure $3(\mathrm{~b})$ shows the emitted power as a function of the load parameter. Our approach shows that the maximum power emitted, even if the small change in velocity is included, is obtained for the absorbed fluxon in contrast to the results in Ref. 3. This difference might be explained by the fact that neither the perturbation theory nor the expression for $\Delta H$ holds in this region. For $R \gg 1$ there is a proportionality between $\Delta H$ and $R^{-1}$. 


\section{CONCLUSION}

We have calculated the dependence of the $I-V$ curve and the emitted power on lossy terminations in long overlapgeometry Josephson junctions. Combination of the results from a perturbation method with an expression for the emitted power valid for $R \gg 1$ yields equations describing the fluxon dynamics in the junction. The perturbation method requires $\alpha \ll 1$ and $l \gg 1$ which in connection with $R \gg 1$ is a parameter range often encountered in experimental situations. Comparison between a numerical experiment and our approach shows reasonable agreement.

Finally, we remark that the phase shift encountered when a fluxon is reflected is not taken into account in our model. However, physical reality requires that $\alpha \ll 1$ such that $u_{\infty} \cong 1$, in this limit the phase shift is approximately given by $2 \sqrt{2}(1-u)^{3 / 2}$

${ }^{1}$ T. V. Rajeevakumar, IEEE Trans. Magn. MAG-17, 591 (1981).

'S. N. Erné and R. D. Parmentier, J. Appl. Phys. 51, 5025 (1980)

${ }^{3}$ S. N. Erné and R. D. Parmentier, J. Appl. Phys. 52, 1608 (1981).

${ }^{4}$ See, e.g., P. S. Lomdahl, "Soliton dynamics in nonintegreable sine-Gordon Systems", DCAMM Report No. S20 (1982), the Technical University of Denmark, and references therein.

${ }^{5}$ O. A. Levring, N. F. Pedersen, and M. R. Samuelsen, Appl. Phys. Lett. 40, $846(1982)$.

${ }^{6}$ O. A. Levring, N. F. Pedersen, and M. R. Samuelsen, J. Appl. Phys. (to be published).

${ }^{7}$ P. L. Christiansen and O. H. Olsen, Physica D 1, 412 (1980).

${ }^{8}$ M. Salerno, Phys. Lett. A 87, $116(1981)$

${ }^{9}$ D. W. McLaughlin and A. C. Scott, Phys. Rev. A 18, 1652 (1978). 